\title{
Association of malalignment, muscular dysfunction, proprioception, laxity and abnormal joint loading with tibiofemoral knee osteoarthritis - a systematic review and meta-analysis
}

\author{
Joyce A. C. van Tunen ${ }^{1 *}$ D, Andrea Dell'Isola ${ }^{2}$, Carsten Juhl ${ }^{1,3}$, Joost Dekker ${ }^{4}$, Martijn Steultjens²,
}

Jonas B. Thorlund ${ }^{1}$ and Hans Lund ${ }^{5}$

\begin{abstract}
Background: To investigate (1) the association of specific biomechanical factors with knee osteoarthritis and knee osteoarthritis development, and (2) the impact of other relevant risk factors on this association.

Methods: MEDLINE, EMBASE, CINAHL and SPORTDiscus were searched up until April 2017. Studies were included if they fulfilled the following criteria: the study 1) assessed the association of a biomechanical factor with knee osteoarthritis, or knee osteoarthritis development; 2) reported on skeletal malalignment, muscular dysfunction, impaired proprioception, laxity and abnormal loading during gait; 3) was a cohort study with participants developing knee osteoarthritis and participants not developing knee osteoarthritis, or a case-control or cross-sectional study with participants with knee osteoarthritis and without knee osteoarthritis. Risk of bias was assessed with the QUIPS tool and meta-analyses were performed using random effects models.
\end{abstract}

Results: Of 6413 unique studies identified, 59 cross-sectional studies were eligible for meta-analyses (9825 participants, 5328 with knee osteoarthritis). No cohort studies fulfilled the inclusion criteria. Compared with healthy controls, patients with knee osteoarthritis have higher odds of having lower muscle strength, proprioception deficits, more medial varusvalgus laxity and less lateral varus-valgus laxity. Patients with medial knee osteoarthritis have higher odds of having a higher knee adduction moment than healthy controls. Level of evidence was graded as 'very low' to 'moderate' quality. Due to large between study differences moderation of other risk factors on biomechanical risk factors could not be evaluated.

Conclusions: Patients with knee osteoarthritis are more likely to display a number of biomechanical characteristics. The causal relationship between specific biomechanical factors and the development of knee osteoarthritis could not be determined as no longitudinal studies were included. There is an urgent need for high quality, longitudinal studies to evaluate the impact of specific biomechanical factors on the development of knee osteoarthritis.

Trial Registration: (PROSPERO ID: CRD42015025092).

Keywords: Osteoarthritis, Knee, Biomechanics, Systematic review, Meta-analysis

\footnotetext{
* Correspondence: jvtunen@health.sdu.dk

${ }^{1}$ Research Unit for Musculoskeletal Function and Physiotherapy, Department

of Sports Science and Clinical Biomechanics, Faculty of Health Sciences,

University of Southern Denmark, Campusvej 55, 5230 Odense, Denmark

Full list of author information is available at the end of the article
}

(c) The Author(s). 2018 Open Access This article is distributed under the terms of the Creative Commons Attribution 4.0 International License (http://creativecommons.org/licenses/by/4.0/), which permits unrestricted use, distribution, and reproduction in any medium, provided you give appropriate credit to the original author(s) and the source, provide a link to the Creative Commons license, and indicate if changes were made. The Creative Commons Public Domain Dedication waiver (http://creativecommons.org/publicdomain/zero/1.0/) applies to the data made available in this article, unless otherwise stated. 


\section{Background}

Tibiofemoral knee osteoarthritis (OA) is mainly considered a mechanically driven disease [1] and numerous interventions such as braces, insoles and exercise therapy aim at modifying potential biomechanical drivers to prevent development or stall progression [2]. Biomechanical knee joint-related factors that often are subject to research in relation to knee OA are skeletal malalignment, muscular dysfunction, impaired proprioception, laxity and abnormal loading during gait [3]. The association of those biomechanical factors and other relevant risk factors (e.g. age, gender, obesity, knee injury) with knee OA and its onset have been reported in many individual studies. Such studies describing biomechanical factors' association with the presence or development of knee $\mathrm{OA}$ are often used to justify specific research questions but may not necessarily be representative of the available literature. Systematic reviews and meta-analyses give an overview the complete evidence of relevant biomechanical factors and their association with knee osteoarthritis.

Some previous reviews have attempted to summarize the evidence for the association between malalignment, muscular dysfunction, impaired proprioception, and laxity with knee OA. However, several of these reviews are not up-to-date and no attempts were made to estimate the magnitude of association of these biomechanical factors with knee OA [4-8]. Whereas systematic reviews including meta-analyses on knee extensor strength and knee joint loading (i.e. knee adduction moment) have primarily focussed on biomechanical risk factors for onset and progression of knee OA [9-11], only one study has systematically compared gait biomechanics in knee OA patients with controls quantitatively [12]. Thus, it has recently been emphasized that systematic reviews and meta-analysis to investigate the relationship between different biomechanical risk factors and OA should be performed [13].

In this systematic review and meta-analysis we aimed to (1) investigate the association of skeletal malalignment, muscular dysfunction, impaired proprioception, laxity and abnormal loading during gait with knee OA and knee OA development, and (2) to investigate the impact of other relevant risk factors on this association. Such knowledge is important when framing future research questions and designing targeted biomechanical interventions.

\section{Methods}

\section{Registration and ethics}

The protocol for this systematic review and meta-analysis [14] has been published previously and has been registered with the International Prospective Register of Systematic Reviews (PROSPERO ID: CRD42015025092). This systematic review and meta-analysis follows the Cochrane Collaboration guideline for preparing systematic review and meta-analysis and are reported according to the Preferred
Reporting Items for Systematic Reviews and Meta-analyses (PRISMA) guideline [15].

\section{Definition of biomechanical domains}

We defined skeletal malalignment as abnormal alignment between the femur and the tibia in the frontal plane (i.e. varus or valgus alignment) [8]. Muscle dysfunction indicates muscle weakness, loss of muscle endurance or changed muscle activation patterns for the muscles that act on the knee joint $[3,5,6]$. Impaired proprioception refers to deterioration of the ability to detect knee joint position and movement [4]. Laxity is a loss of passive joint stabilisation due to the inability of passive structures in and around the knee (knee ligaments, cruciate ligaments, capsule) to provide an adequate counterbalance to the mechanical forces acting upon the knee during activity [7]. Abnormal loading during gait is often represented by evaluating external knee joint moments or the occurrence of varus or valgus thrust [9].

\section{Search strategy}

MEDLINE, EMBASE, SPORTDiscus, and the Cumulative Index to Nursing and Allied Health Literature Database (CINAHL) were searched from their inception until April 2017. Searches used subject headings (MeSH) and text words related to osteoarthritis, biomechanical factors and study types. The complete search strategy can be found in the study protocol [14].

\section{Study selection}

Studies were included if they fulfilled the following criteria: 1) the study assessed the association of a biomechanical factor with knee OA or incident knee OA development; 2) the biomechanical factor in the study was a knee joint related factor that interacts with the forces, moments and kinematics in and around a synovial joint (e.g. skeletal malalignment, proprioception, etc.); 3) the study was a cohort study with participants developing knee OA and participants not developing knee OA, or the study was a case-control or cross-sectional study with participants with knee OA and without knee OA. Studies were excluded if: 1) the study only included participants with patellofemoral osteoarthritis; 2) the study did not distinguish between hip osteoarthritis and knee OA; 3) study participants underwent treatment such as rehabilitation or surgery; 4) the study did not define knee OA in accordance to the criteria described in the protocol; 5) the study compared knees from the same participant (i.e. compared one knee with, and one knee without osteoarthritis within the same participant); 6) both knees are assessed in patients with bilateral knee OA. A detailed description of the eligibility criteria can be found in the study protocol [14].

Two reviewers (JT, ADI) independently screened eligibility of titles and abstracts of the studies obtained by the 
search. Subsequently, the reviewers used a standardized form to select studies eligible for inclusion in the review based on full text. Consensus was reached by discussion.

\section{Assessment of risk of bias}

Risk of bias was scored independently by two reviewers (JT, ADI) using the Quality In Prognostic Studies (QUIPS) tool [16] as described in the protocol [14]. Six areas of potential study biases were assessed: study participation, study attrition, prognostic factor measurement, study confounding, outcome measurement, and statistical analysis and reporting. Attrition was not applicable for cross-sectional studies.

\section{Data extraction}

Two reviewers (JT, ADI) used a customised, pilot-tested form to extract data from the included studies. The following information was extracted by both reviewers: authors, publication year, number of participants developing and not developing knee OA (in cohort studies) or number of participants in the knee OA and control group (in cross-sectional studies), sex, age, body mass index (BMI in $\mathrm{kg} / \mathrm{m}^{2}$ ), knee injury, knee OA definition, radiographic disease severity, involved compartment and examined biomechanical factor.

For malalignment and thrust measurements, the number of patients and controls with and without malalignment/thrust were extracted. If only the group mean and standard deviation was reported for malalignment, we transformed this into the number of participants with varus (or valgus) alignment and the number of participants with neutral and valgus (or varus) alignment by use of the normal deviation, where more than 1 degree deviation signifies abnormal alignment. For muscular dysfunction, impaired proprioception, laxity and external knee joint moments, group mean and standard deviation were extracted for patients and controls. For all factors, odds ratios were extracted if this was the only available data.

Data was grouped per study design, and into the five biomechanical domains. To allow for comparison, data for each biomechanical factor was further divided. Skeletal malalignment was subdivided into varus and valgus alignment, and muscle dysfunction was subdivided into extensor and flexor weakness. Impaired proprioception was subdivided into reposition error, and thresholds to detect a passive movement in the sagittal or frontal plane (varus and valgus direction). Laxity was subdivided into varus-valgus laxity measured at the medial and lateral side, and anterior-posterior laxity. Abnormal loading was subdivided into varus thrust, valgus thrust, knee adduction moment (KADM), knee flexion moment (KFM), etc.. One outcome measure per study for knee extensor and flexor strength was selected based on hierarchies described by Hall et al. [17] and Øiestadt et al.
[10]. Measurements of abnormal loading during gait had to be reported as the peak or maximal value, or had to be examined during the same time period of the gait cycle. Varus and valgus thrust were assessed during early stance. KADM was assessed during early stance or midstance. KFM was assessed during midstance, while knee extension moment (KEM) was assessed during terminal stance.

\section{Statistical analysis}

Mean and standard deviation on continuous scales were transformed to odds ratio (OR) using the Chinn formula as described in the Cochrane Handbook [18]. Meta-analyses using random effects models were applied on the (logarithmic transformed) OR of developing knee OA in participants with the biomechanical factor of interest (cohort studies), or the (logarithmic transformed) OR of the biomechanical factor being present in participants with knee OA compared to the control group (cross-sectional studies). Meta-regression analyses were used to assess the impact of other risk factors (i.e. age, gender, BMI, knee injury) and radiographic severity on the association of biomechanical risk factors with knee OA or knee OA development.

Heterogeneity between studies was examined with standard Q-tests, and calculated as the $\mathrm{I}^{2}$ statistics. Secondary analyses were described in the protocol [14]. The Grading of Recommendation, Assessment, Development and Evaluation (GRADE) framework was used to evaluate quality of evidence [19].

\section{Results}

A total of 6413 unique studies were identified. Eighty-six studies met all eligibility criteria and 59 were included in meta-analyses, as 27 studies did not provide sufficient data for the meta-analysis (Fig. 1).

Only cross-sectional studies were included, as no longitudinal studies fulfilled the in- and exclusion criteria. Therefore, we could not report on the association of specific biomechanical factors and knee OA development. Due to sparse data for most meta-analyses it was not feasible to investigate the impact (moderation) of other relevant risk factors on any associations, thus no meta-regression analyses were performed. Few studies reported data on biomechanical outcomes for different level of radiographic severity, precluding any sub group analysis based on disease severity [20-23]. Therefore, we present a comprehensive overview of the direction and magnitude of associations between knee $\mathrm{OA}$ and biomechanical factors. Duplicate data extraction on 86 studies was deemed not reasonable time wise, thus we performed duplicate data extraction on half of the studies (JT/ADI), and single data extraction on the other half (JT). 


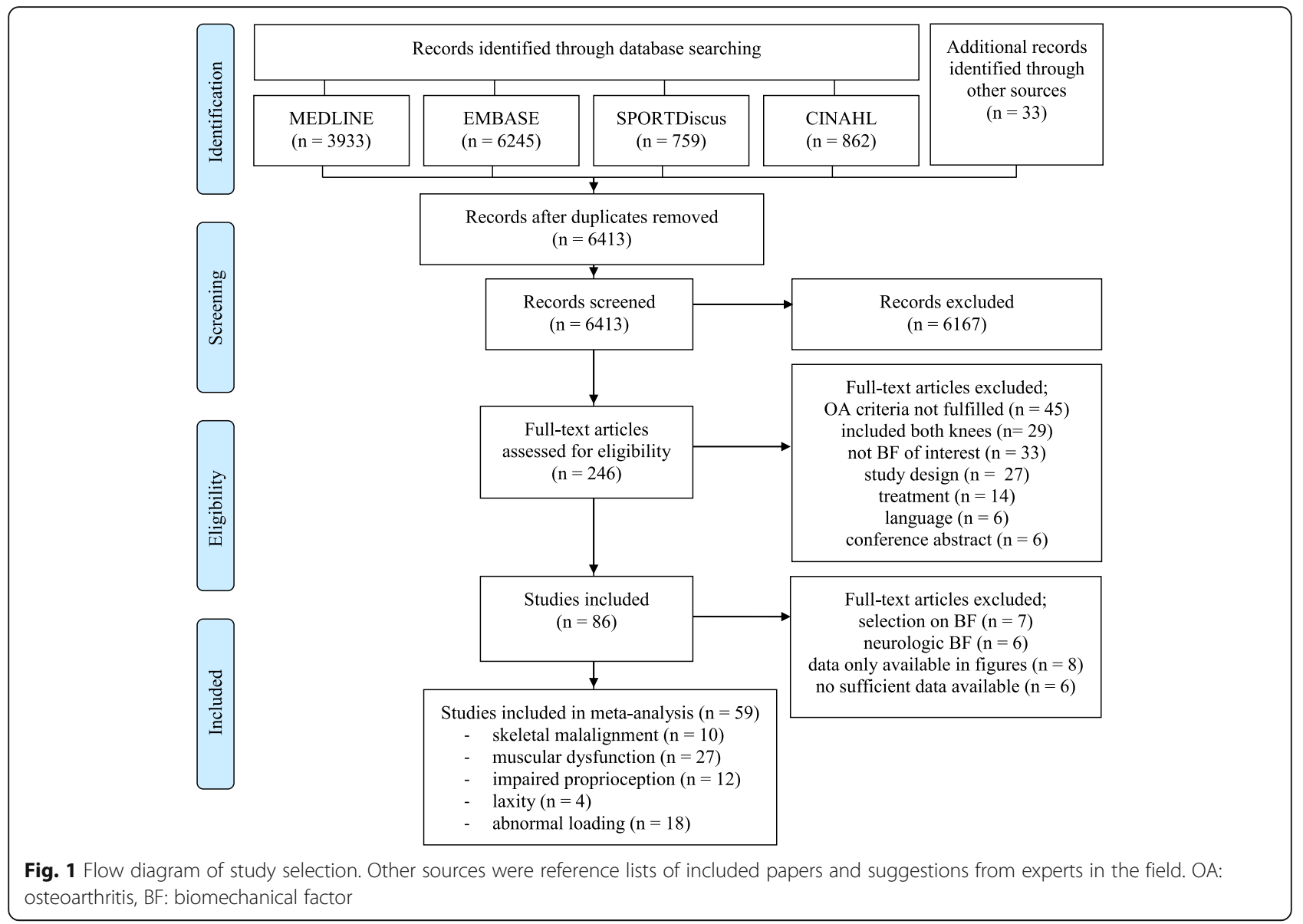

\section{Study characteristics}

The 59 cross-sectional studies for meta-analyses included a total of 9825 participants (5328 patients with knee OA and 4497 healthy controls). 57\% of the knee OA patients and controls were women. Mean age was 63.5 and 61.4 years for knee OA patients and controls, respectively. Mean BMI $\left(\mathrm{kg} / \mathrm{m}^{2}\right)$ was 29.1 for knee OA patients and 26.5 for controls. As few studies included information on previous knee injury, and knee injury was often used as an exclusion criterion for knee OA patients, healthy controls, or both, we have not included information on this. Sixteen studies reported radiographic severity. Twenty-one studies assessed patients with medial knee OA, 37 with a combination of both compartments or the involved compartment was not specified, and one study assessed patients with medial and lateral knee OA separately.

Skeletal malalignment was assessed in 10 studies ( $n=1051)$ [24-33], muscular dysfunction in 27 studies $(n=6086)[20,21,23-25,27,34-54]$, impaired proprioception in 12 studies $(n=565)$ [34, 37, 46, 54-62], laxity in four studies $(n=321)$ [22, 23, 27, $63]$, and abnormal loading in 18 studies $(n=5974)$
[25, 27, 50, 64-78] (Table 1). A summary of findings table can be found in Additional file 1, and forest plots of data pooling for each of the domains and the presence of knee OA can be found in Additional file 2.

As the number of included studies for each specific biomechanical factor was lower than expected, we could not perform all secondary analyses suggested in the protocol. We stratified analyses in the domains malalignment and abnormal loading during gait for medial knee OA, lateral knee OA, and medial/lateral knee OA (a combination of both or involved compartment was not reported), as biomechanical mechanisms will differ based on the involved compartment. The involved compartment was assumed to be medial (or lateral) when $80 \%$ or more of the participants was reported to have medial (or lateral) knee OA.

\section{Assessment of risk of bias of individual studies and overall quality of evidence}

Based on the assessment of risk of bias of individual studies, 32 had a low risk of bias, and 27 studies had a high risk of bias (Table 1). High risk of bias was most often based on a high risk in the areas participation and/ 


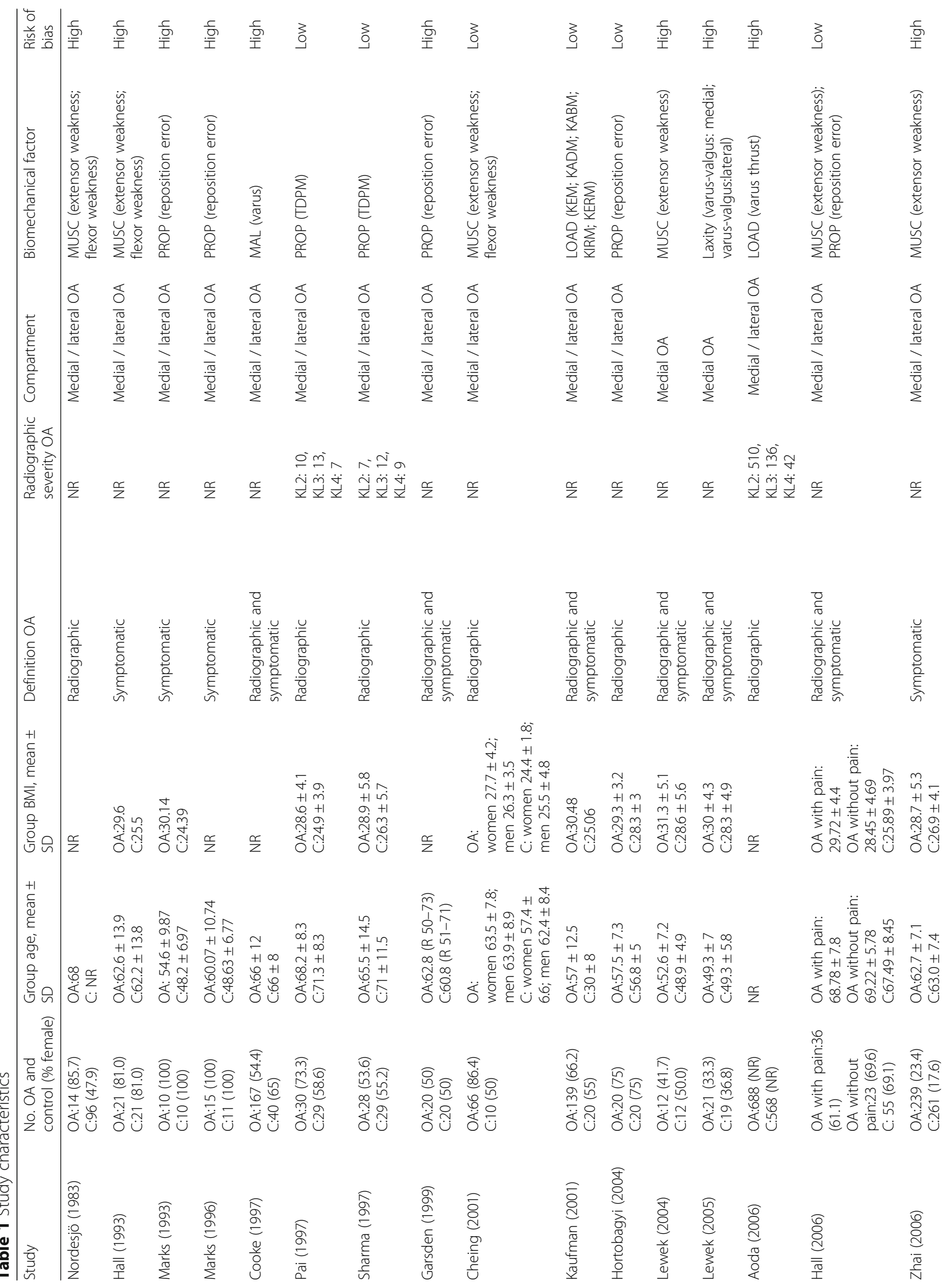




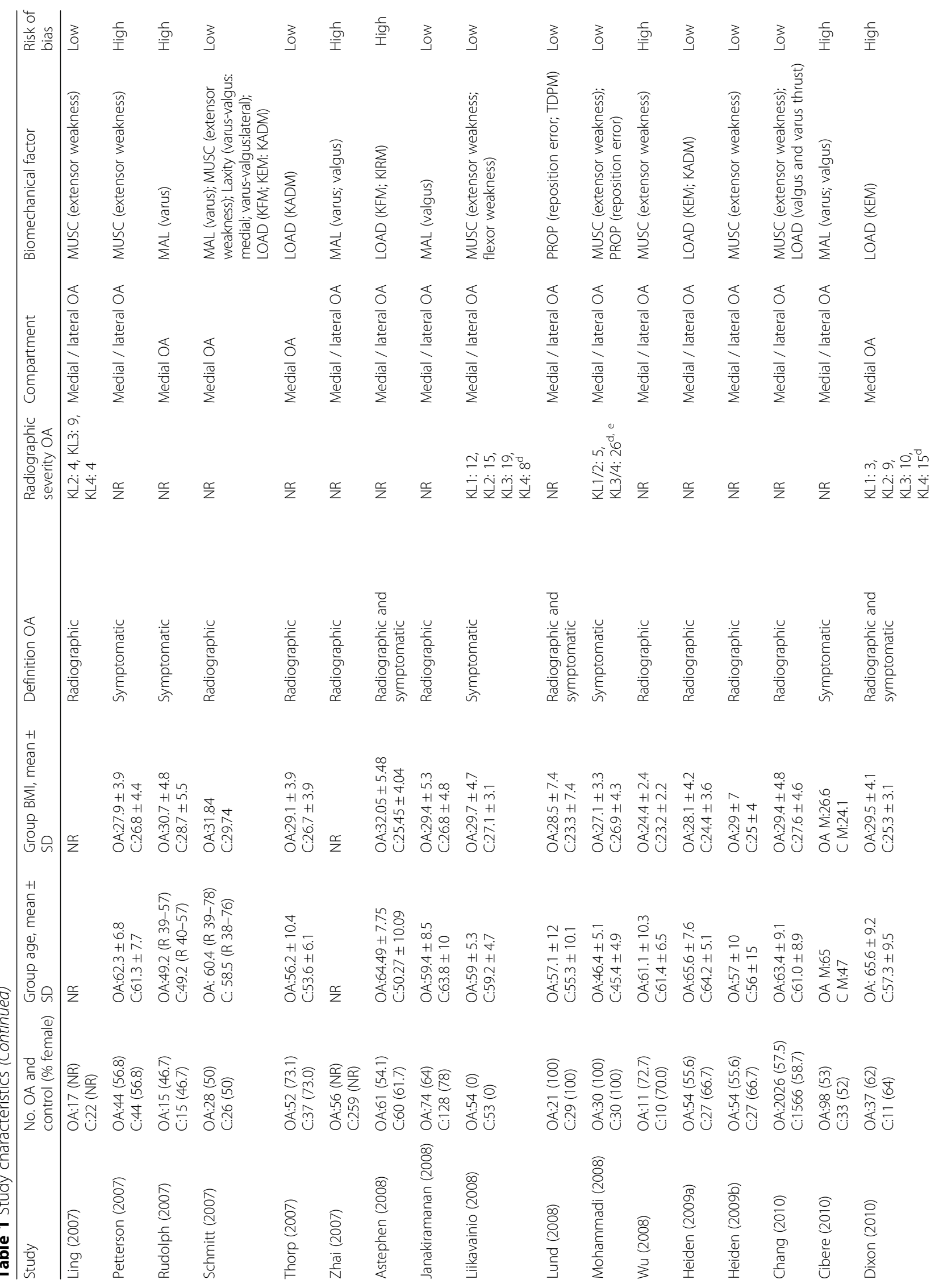




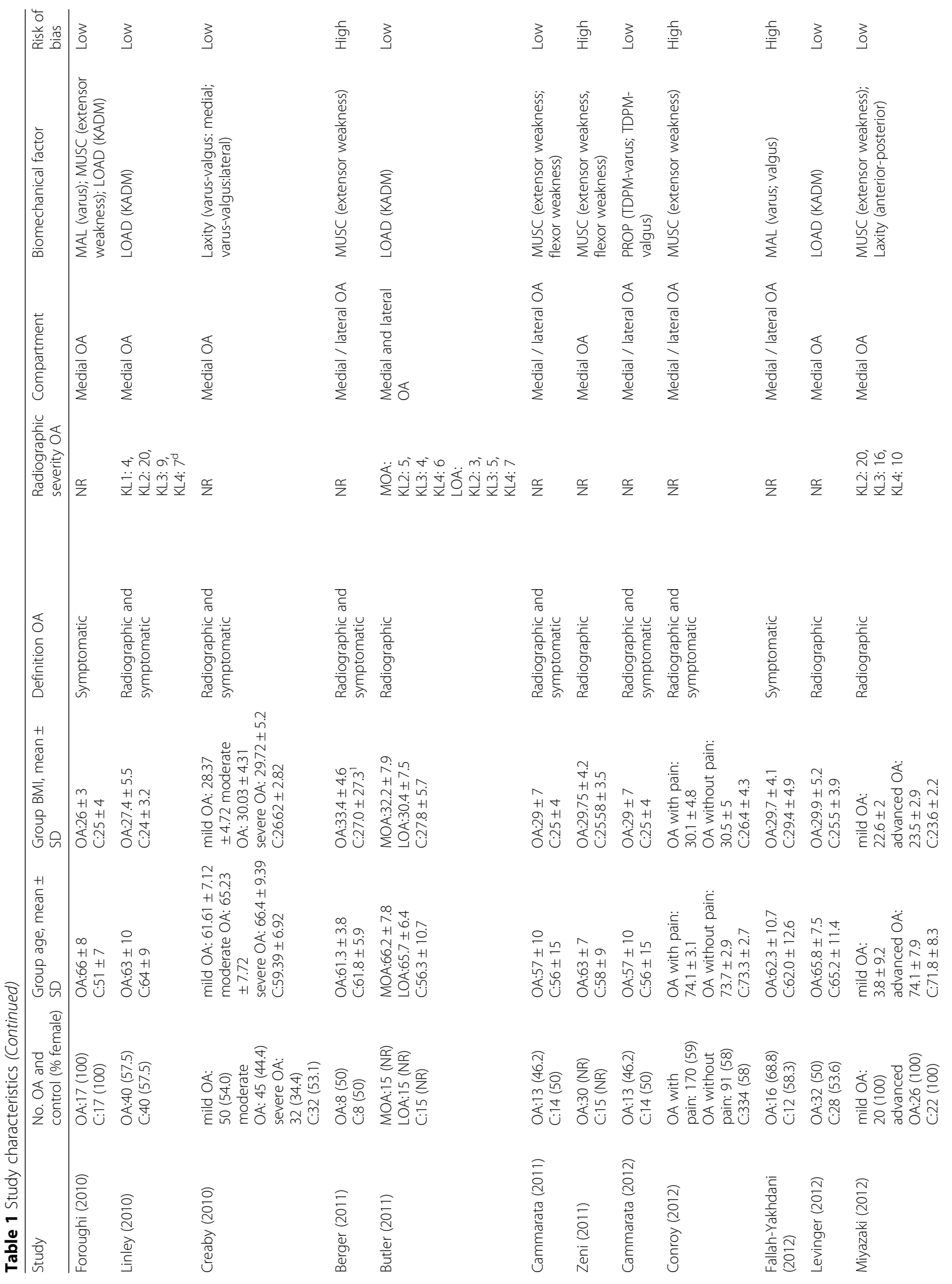




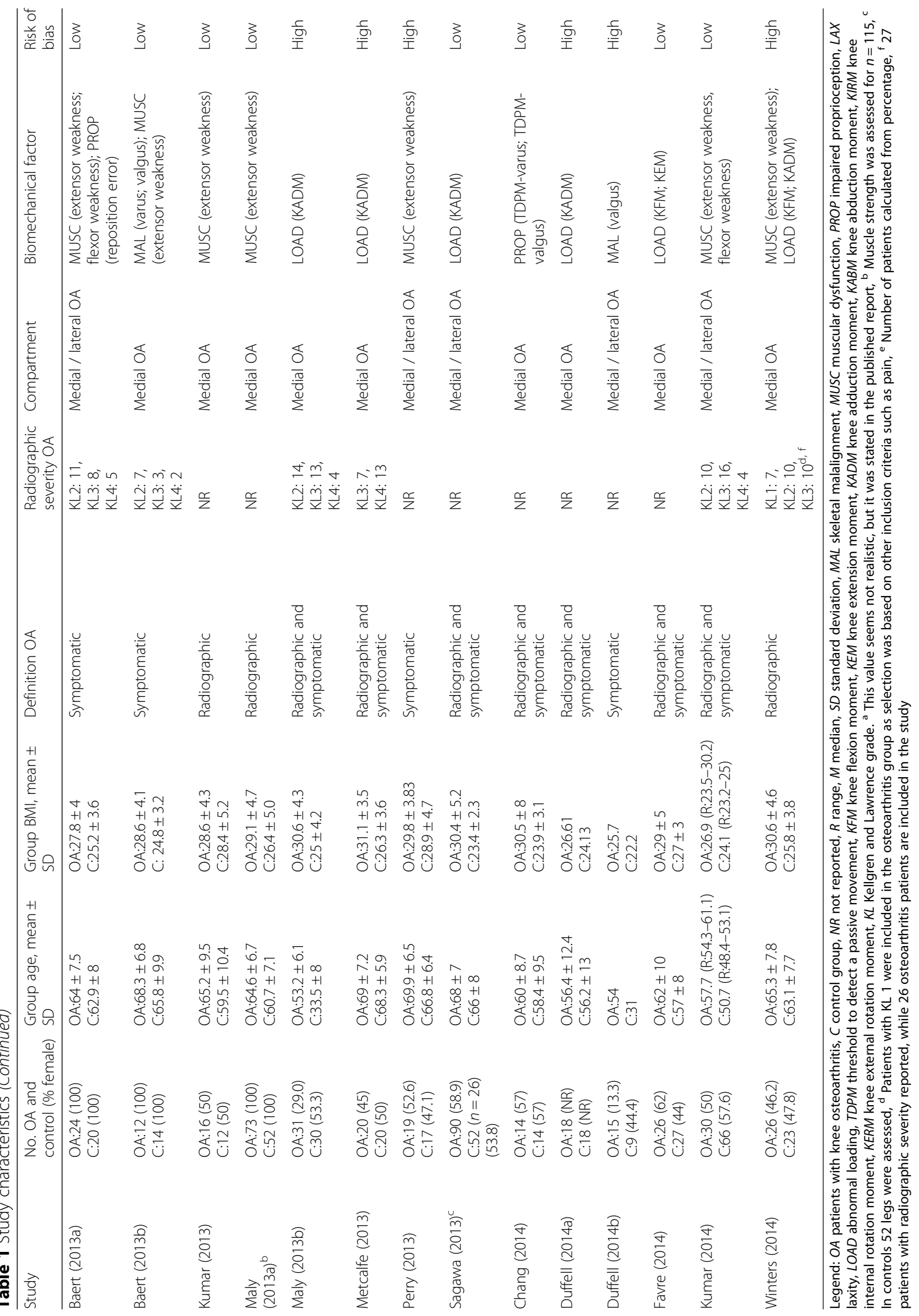


or outcome measurement. Details regarding each of the six areas of potential study biases can be found in Additional file 3.

\section{Skeletal malalignment}

Low quality evidence was found for the odds of having malalignment in patients with knee OA compared to healthy controls (Fig. 2). Varus malalignment, assessed in four studies examining patients with medial knee OA [24-27], and in four studies examining patients with medial and/or lateral knee OA [28-31], was as prevalent in patients with and without knee OA (medial knee OA: $\mathrm{OR}=0.64$ [95\% CI 0.21, 1.97], medial/lateral knee OA: $\mathrm{OR}=0.85$ [95\% CI 0.54, 1.32]). Five studies assessing valgus malalignment in patients with medial and/or lateral knee OA showed no higher odds of having valgus malalignment in patients with knee OA $(\mathrm{OR}=0.80$ [95\% CI $0.40,1.61])[28,30-33]$. One study indicated that patients with knee OA have lower odds of having valgus malalignment compared with healthy controls $(\mathrm{OR}=0.16$ [95\% CI $0.07,0.37])[24]$.

\section{Muscular dysfunction}

Low quality evidence was found for the odds of having muscle weakness in patients with knee OA compared to controls (Fig. 3). Studies assessing muscle weakness showed that patients with medial and/or lateral knee OA had four times higher odds of having muscle weakness compared with healthy controls, both for extensor [21, 23-25, 27, 3454] $\left(\mathrm{OR}=4.02\right.$ [95\% CI 2.69, 6.00], $\mathrm{I}^{2}=89.6 \%$, number of studies $(\mathrm{k})=27)$ and flexor muscles $[21,34,36,38,40,43$,
47, 52] $\left(\mathrm{OR}=4.09\right.$ [95\% CI 1.48, 11.34], $\left.\mathrm{I}^{2}=86.8 \%, \mathrm{k}=8\right)$. Both analyses showed considerable heterogeneity. Data regarding neurological characteristics (i.e. co-contraction index) had also been extracted, but differences in outcome measures and measurement techniques made it impossible to combine these.

\section{Impaired proprioception}

Low to moderate quality evidence was found for the odds of having impaired proprioception in patients with knee OA compared to controls (Fig. 4). Eight studies assessing proprioception as reposition error showed that patients with knee OA have higher odds of having higher reposition error (i.e. poorer proprioception) than healthy controls, with substantial heterogeneity $(\mathrm{OR}=3.26$ [95\% CI 1.73, 6.13], $\left.\mathrm{I}^{2}=63.1 \%\right)[34,46,54-59]$. The odds of having a higher threshold to detect a passive movement in the sagittal plane was higher in patients with knee $\mathrm{OA}(\mathrm{OR}=4.44$ [95\% CI 2.78, 7.10], $\mathrm{I}^{2}=0.0 \%, \mathrm{k}=3$ ), indicating poorer proprioception [57, 60, 62]. Two studies examining knee varus-valgus proprioceptive acuity (i.e. in the frontal plane) showed that patients with knee OA are more likely to have higher threshold to detect a passive movement in the varus direction $\left(\mathrm{OR}=5.29\right.$ [95\% CI 2.00, 13.97], $\left.\mathrm{I}^{2}=0.0 \%\right)$, again indicating poorer proprioception [37, 61]. This was not observed in the valgus direction $(\mathrm{OR}=4.65[95 \% \mathrm{CI} 0.55$, $\left.39.70], \mathrm{I}^{2}=78.9 \%\right)$.

\section{Joint laxity}

All studies assessing joint laxity described patients with medial knee OA (Fig. 5). Three studies reported that

\section{Malalignment}

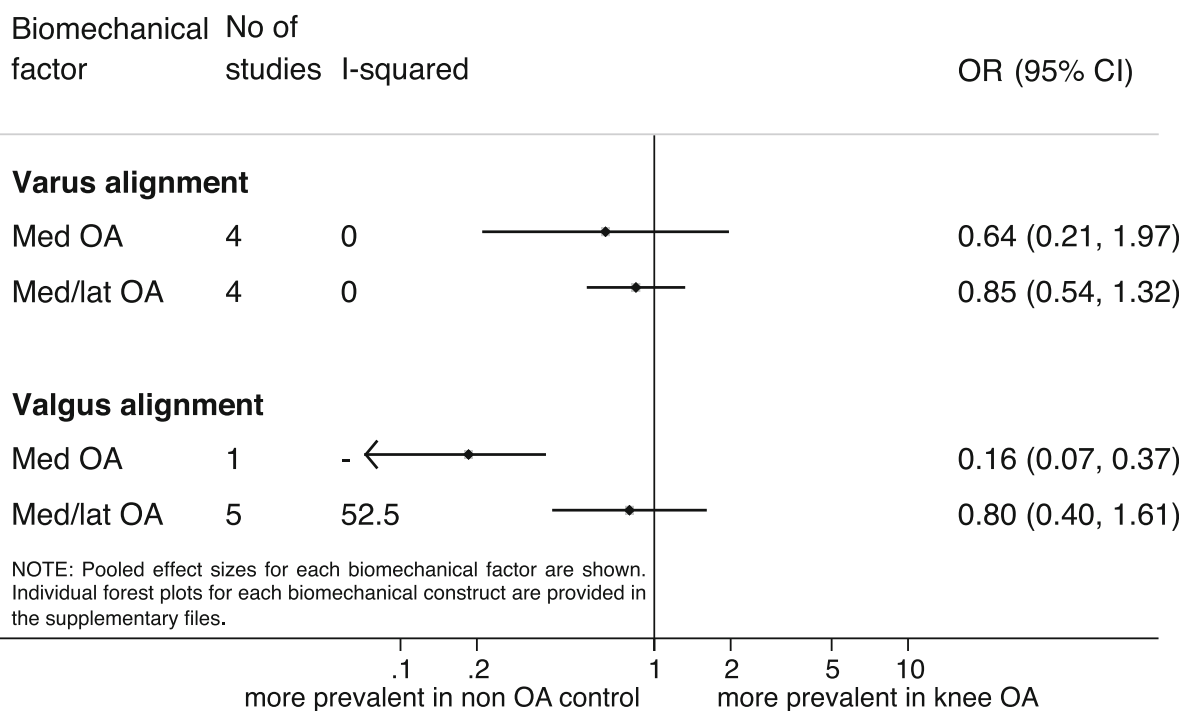

Fig. 2 Results of meta-analyses on skeletal malalignment and the presence of knee osteoarthritis. Results stratified for medial knee OA (Med OA) and a combination of both medial and lateral knee OA (or involved compartment not reported (Med/lat OA)) 


\section{Muscle dysfunction}

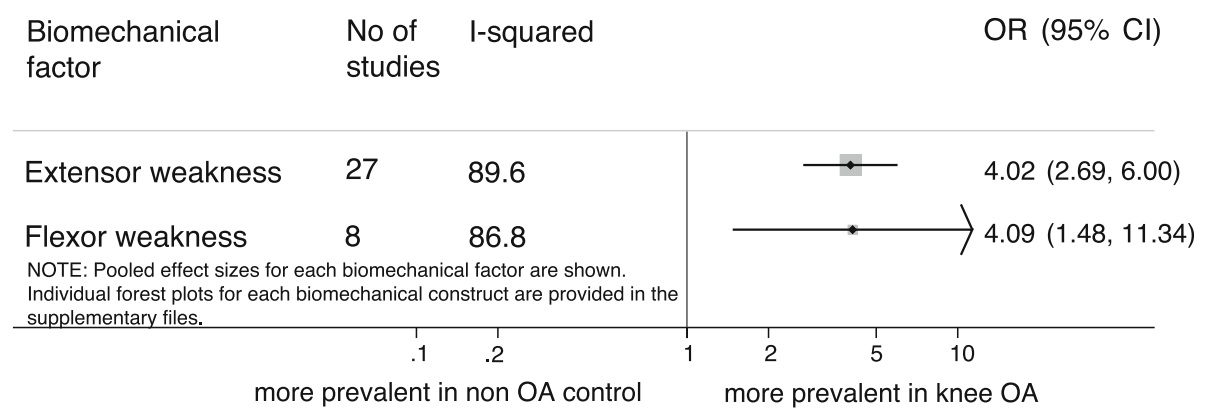

Fig. 3 Results of meta-analyses on muscular dysfunction and the presence of knee osteoarthritis

patients with medial knee OA have four times higher odds of having laxity in varus-valgus direction measured at the medial side of the joint $(\mathrm{OR}=4.23$ [95\% CI 1.34, $\left.13.36], \mathrm{I}^{2}=77.0 \%\right)[22,27,63]$, however, evidence was of low quality and considerable heterogeneity was present. The same three studies reported that patients with medial knee OA have lower odds of having varus-valgus laxity measured at the lateral side of the joint (OR $=0.42[95 \%$ CI $\left.0.25,0.69], \mathrm{I}^{2}=0.0 \%\right)$. Evidence was of low quality. The only study that assessed laxity in anterior-posterior direction suggested no higher or lower odds for patients with medial knee OA compared to healthy controls [23].

\section{Abnormal loading during gait}

Very low to low quality evidence was found for most odds of having abnormal loading in patients with medial and/or lateral knee OA, only moderate quality evidence was found for the odds of having a higher KADM in patients with medial knee OA (Fig. 6). The two studies examining thrust concerned a population with medial and/or lateral knee OA. The odds of having varus thrust was higher in knee OA patients $(\mathrm{OR}=1.46$ [95\% CI 1.00, $\left.2.13], \mathrm{I}^{2}=79.2 \%\right)[64,65]$, while patients with knee OA had no higher odds of having valgus thrust [65]. The odds of having a higher KFM was not higher in patients with medial knee OA [27, 50, 67], while significant higher odds were found for a higher KFM in the healthy controls in one study compared with patients with medial/lateral knee OA [66]. The odds for the presence of a higher knee extension moment (KEM) were not higher in patients with medial and/or lateral knee OA, with considerable heterogeneity [27, 67-70]. Ten studies showed that patients with medial knee OA had higher odds of having a higher knee adduction moment $(\mathrm{KADM})\left(\mathrm{OR}=3.01\right.$ [95\% CI 1.87, 4.85], $\left.\mathrm{I}^{2}=55.5 \%\right)$ [25, $27,50,71-75,77,78]$. The only study [71] assessing this in patients with lateral knee OA reported higher odds for having higher KADM values in healthy controls, and

\section{Impaired proprioception}

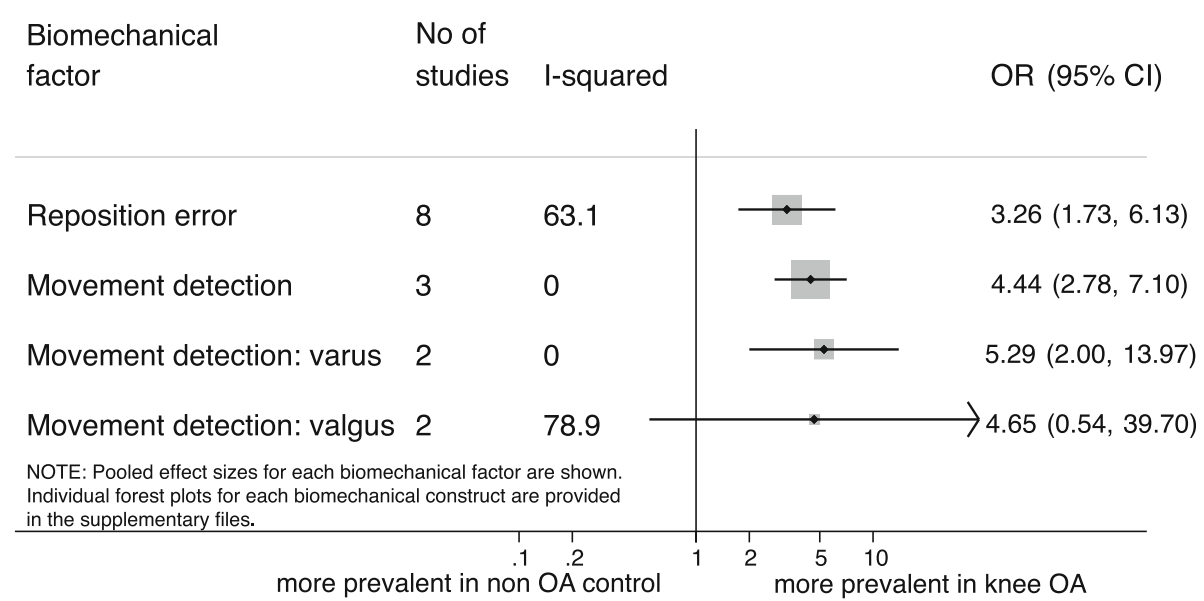

Fig. 4 Results of meta-analyses on impaired proprioception and the presence of knee osteoarthritis. Movement detection refers to the threshold to detect a passive movement in the sagittal or frontal plane 


\section{Laxity}

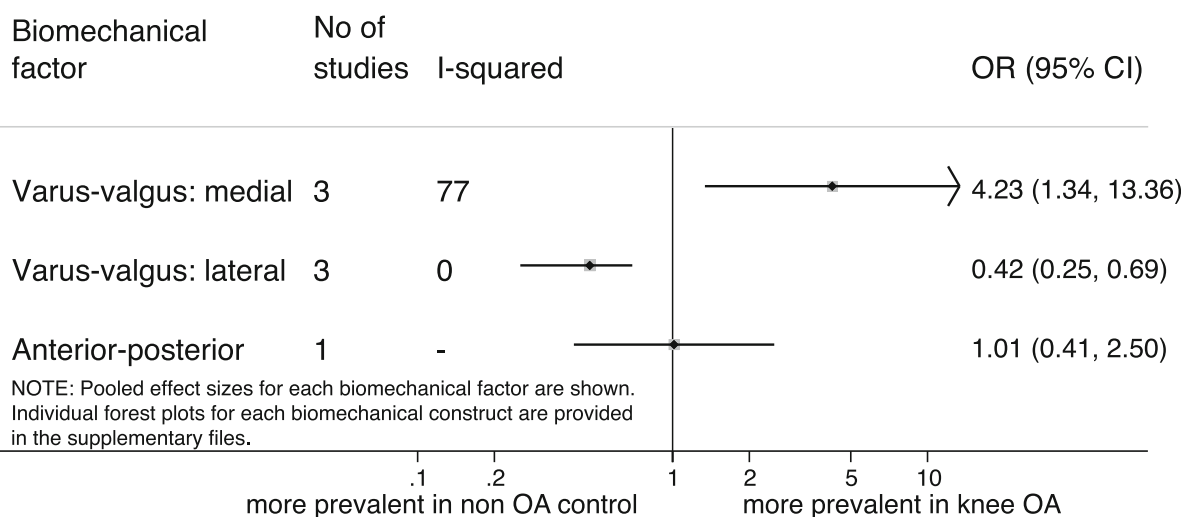

Fig. 5 Results of meta-analyses on laxity and the presence of knee osteoarthritis. Varus-valgus laxity is measured at the medial and lateral side of the knee

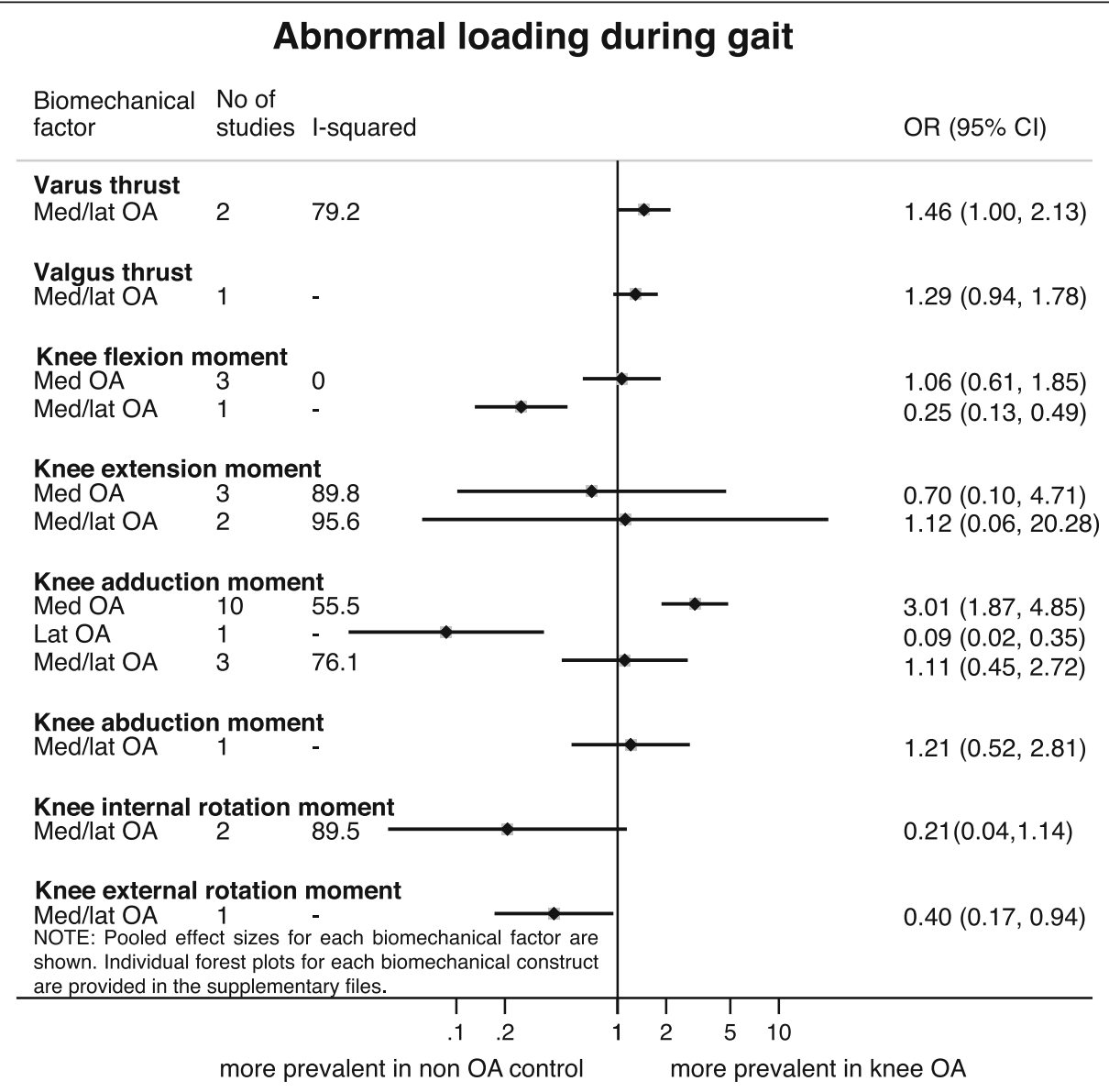

Fig. 6 Results of meta-analyses on abnormal loading during gait and the presence of knee osteoarthritis. Results stratified for medial knee OA (Med OA), lateral knee OA (Lat OA), and a combination of both medial and lateral knee OA (or involved compartment not reported (Med/lat OA)) 
patients with medial/lateral knee OA had no higher or lower odds than healthy controls $(\mathrm{OR}=1.11$ [95\% CI $\left.0.45,2.72], \mathrm{I}^{2}=76.1 \%, \mathrm{k}=3\right)[69,70,76]$. One study assessing both knee abduction moment (KABM) and knee external rotation moment (KERM) showed only higher odds in patients with medial/lateral knee OA for having a higher KERM [70]. The odds for having a higher knee internal rotation moment (KIRM) was not higher in patients with medial and/or lateral knee OA compared to healthy controls $(\mathrm{OR}=0.21$ [95\% CI 0.04, $\left.1.14], \mathrm{I}^{2}=89.5 \%, \mathrm{k}=2\right)[66,70]$.

\section{Discussion}

This systematic review and meta-analyses aimed at investigating the association of specific biomechanical factors with knee OA and knee OA development, and potential effect modification of other relevant risk factors on this association. Based on the predefined selection criteria we did not include any longitudinal studies and sparse data combined with variations in studies for most meta-analyses made precluding any assessment of other relevant risk factors. As a result we could not investigate the association between biomechanical factors and knee OA development, and the impact of other relevant risk factors on any such associations. Thus, this study provides a comprehensive overview of the direction and magnitude of associations between different biomechanical factors and presence of knee OA. Based on mainly low quality evidence following GRADE, the results from this review indicate that patients with knee OA are characterized by lower knee extensor/flexor muscle strength, proprioception deficits, more medial varus-valgus laxity, less lateral varus-valgus laxity, and more varus thrust than healthy controls. Further, patients with medial knee OA are more likely to have a high knee adduction moment compared with healthy controls.

We intended to include longitudinal cohort studies to assess biomechanical risk factors for development of knee OA. However, based on our relative strict inclusion criteria allowing only studies where healthy individuals were available as comparators for assessing biomechanical risk factors, we were not able to include any cohort studies in this review. This was decided because changes in one leg may potentially impact the contralateral leg $[4,57,79]$.

Impaired biomechanics is considered to be an important component of knee OA and such characteristics are often the justification of specific research questions. Although there is considerable evidence from individual studies describing those factors, we could not identify any attempts to summarize the association of skeletal malalignment, muscular dysfunction, impaired proprioception and laxity with knee OA by providing pooled estimates of associations. Pooled estimates have only been presented for the association of abnormal loading with knee OA [12]. This study expands this knowledge by providing an overview of the evidence of which biomechanical attributes describe patients with knee OA.

Our findings that decreased extensor/flexor strength and impaired proprioception are associated with knee OA confirm results of earlier narrative reviews [4-6, 80]. In addition, Freisinger et al. also found that patients with medial knee OA have an increased medial, but not lateral laxity [7]. Biomechanical characteristics during level walking have been the subject of a systematic review and meta-analysis by Mills et al. [12]. In agreement with our study, Mills reported conflicting evidence for the association of KFM with knee OA, which might be due to adaptations of walking pattern to reduce pain and instability [81]. In the same study, evidence for an association of KADM with knee OA was found to be inconclusive, whereas we found that patients with medial knee osteoarthritis have a higher KADM. Lack of stratification for the involved joint compartment in the study by Mills and co-workers could influence the results, as biomechanical mechanisms are expected to differ depending on the involved compartment. We were not able to stratify our varus and valgus thrust analysis for the involved compartment. In combination with the low number of included studies for those factors, this might explain why we only found a borderline significant association for varus thrust with knee OA.

Skeletal malalignment is also commonly reported to be present in patients with knee OA. Surprisingly, this was not found in the present study, although evidence was of low quality and methods used to assess malalignment were not uniform. Several individual studies have reported that varus and/or valgus malalignment are risk factors for the development and/or progression of knee OA [82-84], although one study stated that malalignment is not associated with knee OA development, and suggested that it rather is a marker of disease severity or its progression [85]. Those studies were not included in this review. The main reason for excluding studies was that they compared between knees instead of persons (i.e. allowing the contra-lateral leg as control), as evidence suggests that biomechanical factors are also altered in the contralateral knee highlighting the importance of an independent comparator [4, 57]. Another approach for future attempts to summarize such evidence, could be to include all available data and investigate the importance of type of control (i.e. healthy controls or contra-lateral leg) in sensitivity analysis to also be able to include more longitudinal studies. A systematic review and meta-analysis showed limited evidence for an association between knee malalignment and incident knee OA, although it also showed a relationship between varus and valgus alignment and structural progression of knee OA [8]. 
Limitations of this study warrant consideration. Our findings should be interpreted with caution due to the small number of included studies and the small sample size for most of the biomechanical factors (in particular skeletal malalignment and laxity). As a consequence of the above, and because all studies had a cross-sectional design, we rated most of the quality of evidence to low. Future studies should aim to improve the quality of the evidence.

This systematic review and meta-analysis identified several biomechanical characteristics of patients with knee OA. These findings are important for clinicians, as identification of such biomechanical impairments may help clinicians to better tailor interventions to the individual patient. In fact, the specific biomechanical profile of one patient may mean that some biomechanical interventions could be beneficial and others ineffective or even harmful. Clinical practices may not have the necessary specialized equipment to perform the biomechanical measurements that were performed in the included studies. However, clinical proxy measures exist to examine many of these biomechanical impairments (e.g. manual assessment of laxity or visual assessment of loading during gait by varus or valgus thrust). In addition, longitudinal cohort studies are needed to evaluate the importance of biomechanical factors in the development of knee osteoarthritis. Those studies should aim to evaluate biomechanical factors as risk factors for the development of knee OA. Furthermore, they should focus on the identification of subgroups. This will facilitate identification of persons at high risk of developing knee OA, who might want to be engaged in prevention programs. The longitudinal studies should include healthy persons, who have general risk factors for the development of knee OA, but do not have knee OA at baseline. The presence of knee OA should be assessed at baseline and follow up. General risk factors [86] and at least the biomechanical factors we identified as knee OA characteristics should be assessed. Data of healthy individuals should be compared with data of individuals developing knee OA in one or both knees.

\section{Conclusions}

In conclusion, results indicate that patients with knee $\mathrm{OA}$ are more likely to display a number of biomechanical characteristics such as lower muscle strength, proprioception deficits, more medial varus-valgus laxity and less lateral varus-valgus laxity and higher knee adduction moment (medial knee OA only) compared with healthy controls. The causal relationship between biomechanical factors and the development of knee OA could not be determined as no longitudinal studies were included. High quality longitudinal studies are needed to evaluate the impact of biomechanical factors on the development of knee OA.

\section{Additional files}

Additional file 1: Summary of findings table (DOCX $71 \mathrm{~kb}$ ).

Additional file 2: Forest plots of data pooling for skeletal malalignment, muscular dysfunction, impaired proprioception, laxity and abnormal loading, and the presence of knee osteoarthritis (ZIP $1272 \mathrm{~kb}$ ).

Additional file 3: Risk of bias and individual study quality for the included studies (DOCX $73 \mathrm{~kb}$ ).

\section{Abbreviations}

BMI: Body mass index; C: Control group; Cl: Confidence interval; GRADE: Grading of Recommendation, Assessment, Development and Evaluation; $\mathrm{k}$ : Number of studies; KABM: Knee abduction moment; KADM: Knee adduction moment; KEM: Knee extension moment; KERM: Knee external rotation moment; KFM: Knee flexion moment; KIRM: Knee internal rotation moment; KL: Kellgren and Lawrence grade; LAX: Laxity; LOAD: Abnormal loading; M: Median; MAL: Skeletal malalignment; MUSC: Muscular dysfunction; $n$ : Number of persons; NR: Not reported; OA: Osteoarthritis; OR: Odds ratio; PRISMA: Preferred Reporting Items for Systematic Reviews and Meta-analyses; PROP: Impaired proprioception; QUIPS tool: Quality In Prognostic Studies tool; R: Range; SD: Standard deviation; TDPM: Threshold to detect a passive movement

\section{Funding}

The research leading to these results has received funding form the European Union Seventh Framework Programme (FP7-PEOPLE-2013-ITN; KNEEMO) under grant agreement no. 607510. This funding body had no involvement in the study design, interpretation of data, writing the manuscript or the decision to publish the manuscript.

\section{Availability of data and materials}

The datasets used and/or analysed during the current study are available from the corresponding author on reasonable request.

\section{Authors' contributions \\ JT, ADI, CJ, JD, MS, and HL made substantial contributions to conception and design of the study. JT performed the data analysis and drafted the manuscript. JT, $\mathrm{ADI}, \mathrm{CJ}, \mathrm{JBT}, \mathrm{HL}$ contributed to interpretation of the data. All authors contributed to critical revision of the article for important intellectual content, and read and approved the final version. All authors agreed to be accountable for all aspects for the work in ensuring that questions related to the accuracy or integrity of any part of the work are appropriately investigated and resolved.}

Ethics approval and consent to participate Not applicable.

Consent for publication

Not applicable.

Competing interests

The authors declare that they have no competing interests.

\section{Publisher's Note}

Springer Nature remains neutral with regard to jurisdictional claims in published maps and institutional affiliations.

\footnotetext{
Author details

${ }^{1}$ Research Unit for Musculoskeletal Function and Physiotherapy, Department of Sports Science and Clinical Biomechanics, Faculty of Health Sciences, University of Southern Denmark, Campusvej 55, 5230 Odense, Denmark. ${ }^{2}$ School of Health and Life Sciences, Glasgow Caledonian University, Glasgow, Scotland. ${ }^{3}$ Department of Rehabilitation, Copenhagen University Hospital, Herlev and Gentofte, Denmark. ${ }^{4}$ Department of Rehabilitation Medicine and Department of Psychiatry, EMGO Insitute for Health and Care Research, VU University Medical Center, Amsterdam, the Netherlands. ${ }^{5}$ Centre for Evidence Based Practice, Western Norway University of Applied Sciences, Bergen, Norway.
} 


\section{Received: 21 November 2017 Accepted: 18 July 2018}

\section{Published online: 28 July 2018}

\section{References}

1. Brandt KD, Radin EL, Dieppe PA, van de Putte L. Yet more evidence that osteoarthritis is not a cartilage disease. Ann Rheum Dis. 2006;65:1261-4.

2. Saxby DJ, Lloyd DG. Osteoarthritis year in review 2016: mechanics. Osteoarthr Cartil. 2017;25(2):190-8.

3. Bennell KL, Hunt MA, Wrigley TV, Lim BW, Hinman RS. Role of muscle in the genesis and management of knee osteoarthritis. Rheum Dis Clin N Am. 2008;34(3):731-54.

4. Knoop J, Steultjens MP, van der Leeden M, van der Esch M, Thorstensson CA, Roorda LD, Lems WF, Dekker J. Proprioception in knee osteoarthritis: a narrative review. Osteoarthr Cartil. 2011;19(4):381-8.

5. Bennell KL, Wrigley TV, Hunt MA, Lim BW, Hinman RS. Update on the role of muscle in the genesis and management of knee osteoarthritis. Rheum Dis Clin N Am. 2013;39(1):145-76.

6. Roos EM, Herzog W, Block JA, Bennell KL. Muscle weakness, afferent sensory dysfunction and exercise in knee osteoarthritis. Nat Rev Rheumatol. 2011; 7(1):57-63.

7. Freisinger GM, Schmitt LC, Wanamaker AB, Siston RA, Chaudhari AMW. Tibiofemoral osteoarthritis and varus-valgus laxity. J Knee Surg. 2017;30(5): 440-51

8. Tanamas S, Hanna FS, Cicuttini FM, Wluka AE, Berry P, Urquhart DM. Does knee malalignment increase the risk of development and progression of knee osteoarthritis? A systematic review. Arthritis Rheum. 2009;61(4):459-67.

9. Henriksen M, Creaby MW, Lund H, Juhl C, Christensen R. Is there a causal link between knee loading and knee osteoarthritis progression? A systematic review and meta-analysis of cohort studies and randomised trials. BMJ Open. 2014;4(7):e005368.

10. Oiestad BE, Juhl CB, Eitzen I, Thorlund JB. Knee extensor muscle weakness is a risk factor for development of knee osteoarthritis. A systematic review and meta-analysis. Osteoarthr Cartil. 2015;23(2):171-7.

11. Culvenor AG, Ruhdorfer A, Juhl C, Eckstein F, Oiestad BE. Knee extensor strength and risk of structural, symptomatic, and functional decline in knee osteoarthritis: a systematic review and meta-analysis. Arthritis Care Res. 2017:69(5):649-58.

12. Mills K, Hunt MA, Ferber R. Biomechanical deviations during level walking associated with knee osteoarthritis: a systematic review and meta-analysis. Arthritis Care Res. 2013;65(10):1643-65.

13. Zhang W. Risk factors of knee osteoarthritis--excellent evidence but little has been done. Osteoarthr Cartil. 2010;18(1):1-2.

14. van Tunen JA, Dell'Isola A, Juhl C, Dekker J, Steultjens M, Lund H. Biomechanical factors associated with the development of tibiofemoral knee osteoarthritis: protocol for a systematic review and meta-analysis. BMJ Open. 2016;6(6):e011066.

15. Moher D, Liberati A, Tetzlaff J, Altman DG. Preferred reporting items for systematic reviews and meta-analyses: the PRISMA statement. PLoS Med. 2009;6(7):e1000097.

16. Hayden JA, van der Windt DA, Cartwright JL, Côté P, Bombardier C Assessing bias in studies of prognostic factors. Ann Intern Med. 2013;158: 280-6.

17. Hall M, Juhl C, Lund H, Thorlund JB. Knee extensor muscle strength in middle-aged and older individuals undergoing arthroscopic partial meniscectomy: a systematic review and meta-analysis. Arthritis Care Res. 2015;67(9):1289-96.

18. Higgins JPT, Green S: Cochrane Handbook for Systematic Reviews of Interventions. 2011. Version 5.1.0. [http://handbook-5-1.cochrane.org].

19. Guyatt $\mathrm{GH}$, Oxman AD, Schunemann HJ, Tugwell P, Knottnerus A. GRADE guidelines: a new series of articles in the journal of clinical epidemiology. J Clin Epidemiol. 2011;64(4):380-2.

20. Ling SM, Conwit RA, Talbot L, Shermack M, Wood JE, Dredge EM, Weeks MJ, Abernethy DR, Metter EJ. Electromyographic patterns suggest changes in motor unit physiology associated with early osteoarthritis of the knee. Osteoarthr Cartil. 2007;15(10):1134-40

21. Liikavainio T, Lyytinen T, Tyrvainen E, Sipila S, Arokoski JP. Physical function and properties of quadriceps femoris muscle in men with knee osteoarthritis. Arch Phys Med Rehabil. 2008;89(11):2185-94.

22. Creaby MW, Wrigley TV, Lim BW, Bowles KA, Metcalf BR, Hinman RS, Bennell $\mathrm{KL}$. Varus-valgus laxity and passive stiffness in medial knee osteoarthritis. Arthritis Care Res. 2010;62(9):1237-43.
23. Miyazaki T, Uchida K, Wada M, Sato M, Sugita D, Shimada S, Baba H. Anteroposterior and varus-valgus laxity of the knee increase after stair climbing in patients with mild osteoarthritis. Rheumatol Int. 2012;32(9): 2823-8.

24. Baert IAC, Jonkers I, Staes F, Luyten FP, Truijen S, Verschueren SMP. Gait characteristics and lower limb muscle strength in women with early and established knee osteoarthritis. Clin Biomech. 2013;28(1):40-7.

25. Foroughi N, Smith RM, Lange AK, Baker MK, Fiatarone Singh MA, Vanwanseele B. Dynamic alignment and its association with knee adduction moment in medial knee osteoarthritis. Knee. 2010;17(3):210-6.

26. Rudolph KS, Schmitt LC, Lewek MD. Age-related changes in strength, joint laxity, and walking patterns: are they related to knee osteoarthritis? Phys Ther. 2007:87(11):1422-32.

27. Schmitt LC, Rudolph KS. Influences on knee movement strategies during walking in persons with medial knee osteoarthritis. Arthritis Rheum. 2007: 57(6):1018-26

28. Cibere J, Zhang $H$, Thorne A, Wong $H$, Singer J, Kopec JA, Guermazi A, Peterfy C, Nicolaou S, Munk PL, et al. Association of clinical findings with pre-radiographic and radiographic knee osteoarthritis in a population-based study. Arthritis Care Res. 2010;62(12):1691-8.

29. Cooke DT, Scudamore A, Li J, Wyss U, Bryant T, Costigan P. Axial lower-limb alignment; comparison of knee geometry in normal volunteers and osteoarthritis patients. Osteoarthr Cartil. 1997;5:39-47.

30. Fallah-Yakhdani HR, Abbasi-Bafghi H, Meijer OG, Bruijn SM, van den Dikkenberg N, Benedetti M-G, van Dieën JH. Determinants of co-contraction during walking before and after arthroplasty for knee osteoarthritis. Clin Biomech. 2012;27(5):485-94.

31. Zhai G, Ding C, Cicuttini F, Jones G. A longitudinal study of the association between knee alignment and change in cartilage volume and chondral defects in a largely non-osteoarthritic population. J Rheumatol. 2007;34(1): $181-6$.

32. Janakiramanan $\mathrm{N}$, Teichtahl AJ, Wluka AE, Ding C, Jones G, Davis SR, Cicuttini FM. Static knee alignment is associated with the risk of unicompartmental knee cartilage defects. J Orthop Res. 2008;26(2):225-30.

33. Duffell LD, Mushtaq J, Masjedi M, Cobb JP. The knee adduction angle of the osteo-arthritic knee: a comparison of 3D supine, static and dynamic alignment. Knee. 2014;21(6):1096-100.

34. Baert IA, Mahmoudian A, Nieuwenhuys A, Jonkers I, Staes F, Luyten FP, Truijen S, Verschueren SM. Proprioceptive accuracy in women with early and established knee osteoarthritis and its relation to functional ability, postural control, and muscle strength. Clin Rheumatol. 2013;32(9):1365-74.

35. Berger MJ, Chess DG, Doherty TJ. Vastus medialis motor unit properties in knee osteoarthritis. BMC Musculoskelet Disord. 2011;12:199.

36. Cammarata ML, Schnitzer TJ, Dhaher YY. Does knee osteoarthritis differentially modulate proprioceptive acuity in the frontal and sagittal planes of the knee? Arthritis Rheum. 2011;63(9):2681-9.

37. Chang AH, Lee SJ, Zhao H, Ren Y, Zhang LQ. Impaired varus-valgus proprioception and neuromuscular stabilization in medial knee osteoarthritis. J Biomech. 2014;47(2):360-6.

38. Cheing GL, Hui-Chan CW. The motor dysfunction of patients with knee osteoarthritis in a Chinese population. Arthritis Rheum. 2001;45(1):62-8.

39. Conroy MB, Kwoh CK, Krishnan E, Nevitt MC, Boudreau R, Carbone LD, Chen $H$, Harris TB, Newman AB, Goodpaster BH. Muscle strength, mass, and quality in older men and women with knee osteoarthritis. Arthritis Care Res. 2012:64(1):15-21.

40. Hall KD, Hayes KW, Falconer J. Differential strength decline in patients with osteoarthritis of the knee: revision of a hypothesis. Arthritis Care Res. 1993;6(2):89-96

41. Heiden TL, Lloyd DG, Ackland TR. Knee extension and flexion weakness in people with knee osteoarthritis: is antagonist cocontraction a factor? J Orthop Sports Phys Ther. 2009:39(11):807-15.

42. Kumar D, Manal KT, Rudolph KS. Knee joint loading during gait in healthy controls and individuals with knee osteoarthritis. Osteoarthr Cartil. 2013; 21(2):298-305.

43. Kumar D, Karampinos DC, MacLeod TD, Lin W, Nardo L, Li X, Link TM, Majumdar S, Souza RB. Quadriceps intramuscular fat fraction rather than muscle size is associated with knee osteoarthritis. Osteoarthr Cartil. 2014;22(2):226-34

44. Lewek MD, Rudolph KS, Snyder-Mackler L. Control of frontal plane knee laxity during gait in patients with medial compartment knee osteoarthritis. Osteoarthr Cartil. 2004;12(9):745-51. 
45. Maly MR, Calder KM, Macintyre NJ, Beattie KA. Relationship of intermuscular fat volume in the thigh with knee extensor strength and physical performance in women at risk of or with knee osteoarthritis. Arthritis Care Res. 2013;65(1):44-52.

46. Mohammadi F, Taghizadeh S, Ghaffarinejad F, Khorrami M, Sobhani S. Proprioception, dynamic balance and maximal quadriceps strength in females with knee osteoarthritis and normal control subjects. Int J Rheum Dis. 2008;11(1):39-44.

47. Nordesjö L-O, Nordgren B, Wigren A, Kolstad K. Isometric strength and endurance in patients with severe rheumatoid arthritis or Osteoarthrosis in the knee joints. Scand J Rheumatol. 1983;12(2):152-6.

48. Petterson SC, Raisis L, Bodenstab A, Snyder-Mackler L. Disease-specific gender differences among total knee arthroplasty candidates. J Bone Joint Surg A. 2007:89(11):2327-33.

49. Perry BD, Levinger $P$, Serpiello FR, Caldow MK, Cameron-Smith D, Bartlett JR, Feller JA, Bergman NR, Levinger I, McKenna MJ. The effects of osteoarthritis and age on skeletal muscle strength, Na+-K+-ATPase content, gene and isoform expression. J Appl Physiol. 2013;115(10):1443-9.

50. Winters JD, Rudolph KS. Quadriceps rate of force development affects gait and function in people with knee osteoarthritis. Eur J Appl Physiol. 2014;114(2):273-84.

51. Wu S, Chu N, Liu Y, Chen C, Tang SFT, Cheng C. Relationship between the EMG ratio of muscle activation and bony structure in osteoarthritic knee patients with and without patellar malalignment. J Rehabil Med. 2008;40(5):381-6.

52. Zeni JA, Higginson JS. Knee osteoarthritis affects the distribution of joint moments during gait. Knee. 2011;18(3):156-9.

53. Zhai G, Blizzard L, Srikanth V, Ding C, Cooley H, Cicuttini F, Jones G. Correlates of knee pain in older adults: Tasmanian older adult cohort study. Arthritis Care Res. 2006;55(2):264-71

54. Hall MC, Mockett SP, Doherty M. Relative impact of radiographic osteoarthritis and pain on quadriceps strength, proprioception, static postural sway and lower limb function. Ann Rheum Dis. 2006;65(7):865-70.

55. Garsden LR, Bullock-Saxton JE. Joint reposition sense in subjects with unilateral osteoarthritis of the knee. Clin Rehabil. 1999;13:148-55.

56. Hortobagyi T, Garry J, Holbert D, Devita P. Aberrations in the control of quadriceps muscle force in patients with knee osteoarthritis. Arthritis Rheum. 2004;51(4):562-9.

57. Lund $H$, Juul-Kristensen $B$, Hansen $K$, Christensen $R$, Christensen $H$ Danneskiold-Samsoe B, Bliddal H. Movement detection impaired in patients with knee osteoarthritis compared to healthy controls: a cross-sectional case-control study. J Musculoskelet Neuronal Interact. 2008;8(4):391-400.

58. Marks R, Quinney HA, Wessel J. Proprioceptive sensibility in women with normal and osteoarthritic knee joints. Clin Rheumatol. 1993;12(2):170-5.

59. Marks R. Further evidence of impaired position sense in knee osteoarthritis. Physiother Res Int. 1996;1 (2):127-36.

60. Pai YC, Rymer WZ, Chang RW, Sharma L. Effect of age and osteoarthritis on knee proprioception. Arthritis Rheum. 1997:40(12):2260-5.

61. Cammarata ML, Dhaher YY. Associations between frontal plane joint stiffness and proprioceptive acuity in knee osteoarthritis. Arthritis Care Res. 2012;64(5):735-43.

62. Sharma L, Pai YC, Holtkamp K, Rymer WZ. Is knee joint proprioception worse in the arthritic knee versus the unaffected knee in unilateral knee osteoarthritis? Arthritis Rheum. 1997:40(8):1518-25.

63. Lewek MD, Ramsey DK, Snyder-Mackler L, Rudolph KS. Knee stabilization in patients with medial compartment knee osteoarthritis. Arthritis Care Res. 2005;52(9):2845-53.

64. Aoda H, Nakamura K, Omori G, Koga Y, Akazawa K, Yamamoto M. Independent predictors of knee osteoarthritis in an elderly Japanese population: a multivariate analysis. Acta Med Biol. 2006;54(2):33-41.

65. Chang A, Hochberg M, Song J, Dunlop D, Chmiel JS, Nevitt M, Hayes K, Eaton C, Bathon J, Jackson R, et al. Frequency of varus and valgus thrust and factors associated with thrust presence in persons with or at higher risk of developing knee osteoarthritis. Arthritis Rheum. 2010;62(5):1403-11.

66. Astephen JL, Deluzio KJ, Caldwell GE, Dunbar MJ. Biomechanical changes at the hip, knee, and ankle joints during gait are associated with knee osteoarthritis severity. J Orthop Res. 2008;26(3):332-41.

67. Favre J, Erhart-Hledik JC, Andriacchi TP. Age-related differences in sagittalplane knee function at heel-strike of walking are increased in osteoarthritic patients. Osteoarthr Cartil. 2014;22(3):464-71.

68. Dixon SJ, Hinman RS, Creaby MW, Kemp G, Crossley KM. Knee joint stiffness during walking in knee osteoarthritis. Arthritis Care Res. 2010;62(1):38-44.
69. Heiden TL, Lloyd DG, Ackland TR. Knee joint kinematics, kinetics and muscle co-contraction in knee osteoarthritis patient gait. Clin Biomech. 2009;24(10): 833-41.

70. Kaufman KR, Hughes C, Morrey BF, Morrey M, An KN. Gait characteristics of patients with knee osteoarthritis. J Biomech. 2001;34(7):907-15.

71. Butler RJ, Barrios JA, Royer T, Davis IS. Frontal-plane gait mechanics in people with medial knee osteoarthritis are different from those in people with lateral knee osteoarthritis. Phys Ther. 2011;91(8):1235-43.

72. Levinger P, Menz HB, Morrow AD, Feller JA, Bartlett JR, Bergman NR. Foot kinematics in people with medial compartment knee osteoarthritis. Rheumatology. 2012;51(12):2191-8.

73. Linley HS, Sled EA, Culham EG, Deluzio KJ. A biomechanical analysis of trunk and pelvis motion during gait in subjects with knee osteoarthritis compared to control subjects. Clin Biomech. 2010;25(10):1003-10.

74. Metcalfe AJ, Stewart C, Postans N, Dodds AL, Holt CA, Roberts AP. The effect of osteoarthritis of the knee on the biomechanics of other joints in the lower limbs. Bone Jt J. 2013;95(3):348-53.

75. Thorp LE, Sumner DR, Wimmer MA, Block JA. Relationship between pain and medial knee joint loading in mild radiographic knee osteoarthritis. Arthritis Rheum. 2007;57(7):1254-60.

76. Sagawa Y Jr, Armand S, Lubbeke A, Hoffmeyer P, Fritschy D, Suva D, Turcot $K$. Associations between gait and clinical parameters in patients with severe knee osteoarthritis: a multiple correspondence analysis. Clin Biomech. 2013; 28(3):299-305.

77. Duffell LD, Southgate DF, Gulati V, McGregor AH. Balance and gait adaptations in patients with early knee osteoarthritis. Gait Posture. 2014;39(4):1057-61.

78. Maly MR, Robbins SM, Stratford PW, Birmingham TB, Callaghan JP. Cumulative knee adductor load distinguishes between healthy and osteoarthritic knees--a proof of principle study. Gait Posture. 2013;37(3):397-401.

79. Messier SP, Beavers DP, Herman C, Hunter DJ, DeVita P. Are unilateral and bilateral knee osteoarthritis patients unique subsets of knee osteoarthritis? A biomechanical perspective. Osteoarthr Cartil. 2016;24(5):807-13.

80. Wodowski AJ, Swigler CW, Liu H, Nord KM, Toy PC, Mihalko WM. Proprioception and knee Arthroplasty: a literature review. Orthop Clin North Am. 2016:47(2):301-9.

81. Varady NH, Grodzinsky AJ. Osteoarthritis year in review 2015: mechanics. Osteoarthr Cartil. 2016:24(1):27-35.

82. Brouwer GM, Tol AW, Bergink AP, Belo JN, Bernsen RM, Reijman M, Pols HA, Bierma-Zeinstra SM. Association between valgus and varus alignment and the development and progression of radiographic osteoarthritis of the knee. Arthritis Rheum. 2007:56(4):1204-11.

83. Sharma L, Song J, Dunlop D, Felson D, Lewis CE, Segal N, Torner J, Cooke TD, Hietpas J, Lynch J, et al. Varus and valgus alignment and incident and progressive knee osteoarthritis. Ann Rheum Dis. 2010;69(11):1940-5.

84. Felson DT, Niu J, Gross KD, Englund M, Sharma L, Cooke TD, Guermazi A, Roemer FW, Segal N, Goggins JM, et al. Valgus malalignment is a risk factor for lateral knee osteoarthritis incidence and progression: findings from the multicenter osteoarthritis study and the osteoarthritis initiative. Arthritis Rheum. 2013;65(2):355-62.

85. Hunter DJ, Niu J, Felson DT, Harvey WF, Gross KD, McCree P, Aliabadi P, Sack B, Zhang Y. Knee alignment does not predict incident osteoarthritis: the Framingham osteoarthritis study. Arthritis Rheum. 2007;56(4):1212-8.

86. Silverwood V, Blagojevic-Bucknall M, Jinks C, Jordan JL, Protheroe J, Jordan KP. Current evidence on risk factors for knee osteoarthritis in older adults: a systematic review and meta-analysis. Osteoarthr Cartil. 2015;23(4):507-15.

\section{Ready to submit your research? Choose BMC and benefit from:}

- fast, convenient online submission

- thorough peer review by experienced researchers in your field

- rapid publication on acceptance

- support for research data, including large and complex data types

- gold Open Access which fosters wider collaboration and increased citations

- maximum visibility for your research: over $100 \mathrm{M}$ website views per year

At BMC, research is always in progress.

Learn more biomedcentral.com/submission 